\title{
Sustained complete response on crizotinib in primary lung inflammatory myofibroblastic tumor - Case report and literature review
}

\author{
Abhenil Mittal $^{1}$, Aarushi Gupta ${ }^{2}$, Ekta Dhamija ${ }^{3}$, Adarsh Barwad ${ }^{4}$, Sameer Rastogi $^{1}$ \\ ${ }^{1}$ Department of Medical Oncology, Institute Rotary Cancer Hospital, All India Institute of Medical Sciences, New Delhi; \\ ${ }^{2}$ Department of Radiodiagnosis, Atal Bihari Vajpayee Institute of Medical Sciences and Dr. Ram Manohar Lohia \\ Hospital, New Delhi; ${ }^{3}$ Department of Radiodiagnosis, Institute Rotary Cancer Hospital, All India Institute of Medical \\ Sciences, New Delhi; ${ }^{4}$ Department of Pathology, All India Institute of Medical Sciences, New Delhi, India
}

\begin{abstract}
Inflammatory myofibroblastic tumors (IMT) are rare soft tissue tumors of intermediate malignant potential occurring usually in children and adolescents. Treatment options for advanced disease are limited. A 35-year-old lady presented to us with fever, cough and decreased appetite. On evaluation, she was diagnosed with left lung IMT. She underwent surgery and developed recurrence with pleural nodules after two years. Immunohistochemistry showed positivity for ALK (diffuse). Since recent evidence suggested that crizotinib is effective in advanced IMT with $50 \%$ response rates, she was treated with crizotinib $250 \mathrm{mg}$ BD with which she had a complete radiological response at three months. She has completed one year of treatment thus far and continues to be in complete remission. Treatment with ALK inhibitors like crizotinib has brought about a paradigm shift in the management
\end{abstract}

Correspondence: Sameer Rastogi, M.D., D.M., Assistant Professor, Department of Medical Oncology, All India Institute of Medical Sciences, New Delhi 110029, India.

E- mail: samdoc_mamc@yahoo.com

Key words: Crizotinib, inflammatory myofibroblastic tumor, ALK

Contributions: AM, AG, conceptualization, manuscript writing; $\mathrm{AB}$, $\mathrm{ED}$; investigation, final approval; SR, supervision, editing, final approval.

Conflict of interest: The authors declare that they have no competing interests, and all authors confirm accuracy.

Ethics Approval: Not applicable.

Informed consent: Obtained from the patient.

Received for publication: 23 August 2020.

Accepted for publication: 12 January 2021.

${ }^{\circ}$ Copyright: the Author(s), 2021

Licensee PAGEPress, Italy

Monaldi Archives for Chest Disease 2021; 91:1586

doi: 10.4081/monaldi.2021.1586

This article is distributed under the terms of the Creative Commons Attribution Noncommercial License (by-nc 4.0) which permits any noncommercial use, distribution, and reproduction in any medium, provided the original author(s) and source are credited. of advanced ALK-positive IMT's with excellent clinical responses which are durable in a majority of cases.

\section{Highlights}

1. Inflammatory Myofibroblastic Tumor is a rare soft tissue tumor of lung with intermediate malignant potential;

2. $50 \%$ of patients with unresectable tumors have ALK rearrangements;

3. Crizotinib provides durable responses in half of patients with excellent safety profile.

\section{Introduction}

Inflammatory myofibroblastic tumor (IMT), originally a member of the inflammatory pseudotumor family was first described in 1939 in the lung [1]. Since its first description, the understanding of biology and clinical features of IMT has undergone a paradigm change. They are now considered potentially malignant with a propensity for local recurrence and rarely metastasis [2]. Most often presenting as a mass in the abdomino-pelvic region of children and young adults, they have been described in almost all anatomical locations $[3,4]$. Histologically, IMTs are characterized by a variably cellular spindle cell proliferation in a myxoid to collagenous stroma with a prominent inflammatory infiltrate composed primarily of plasma cells and lymphocytes, with occasional admixed eosinophils and neutrophils. Three basic patterns namely myxoid/vascular, spindle cell and hypocellular fibrous were described by Coffin et al in 1995 and are still widely recognized [3]. Immunohistochemistry is often positive for smooth muscle actin in $80-90 \%$ of cases and desmin/calponin in $60-70 \%$ though they are often focal and diagnosis requires recognition of microscopic features by an experienced sarcoma pathologist [3]. Generally, pathological features do not corelate with outcome in IMT except epithelioid variant which is postulated to have an aggressive course and inferior prognosis. As far as Indian literature is concerned, largest single center case series of 6 cases of IMT has been described; three were abdominal, two arising from head and neck and one from cervix [5]. Surgery (open or minimally invasive) remains the treatment of choice for localized resectable disease as described in previous reports from India [6,7].

With the discovery of anaplastic lymphoma kinase (ALK) rearrangements in around 50\% of cases of IMT's, potential for targeted therapy with ALK inhibitors and subsequent reports of effi- 
cacy of crizotinib with responses in around half of ALK positive patients; management of IMT has undergone a paradigm change. Here, we present one such case of young woman with recurrent lung IMT with sustained complete response to crizotinib. To the best of our knowledge, this is the first report from India.

\section{Case Report}

A 35-year-old lady presented in June 2017 with low grade fever, decreased appetite and dry cough for four months. On evaluation, contrast enhanced CT scan of the chest showed a $6.5 * 5 * 5.5$ $\mathrm{cm}$ mass lesion in the left lingular segment abutting mediastinal pleura, left cardiac margin and costal pleural reaching up to hilum (Figure $1 \mathrm{a}, \mathrm{b}$ ). There was no evidence of metastatic disease. Biopsy from the mass was suggestive of spindle cell tumor in a myxoid background with admixed lymphoplasmacytic cells. Tumor cells were positive for ALK (diffuse) and smooth muscle actin (SMA) while being negative for cytokeratin (CK), CD34 and S100 (Figure 2 A-D). Overall it was suggestive of IMT of the Lung. She underwent surgery (minithoracotomy with left lingular mass excision with mesh repair of pericardium) and was kept on observation subsequently. She developed recurrence in February 2019 tiny irregular parenchymal nodule in right lung and two pleural based deposits in left lung (Figure 1c). There was no other site of disease. She was started on crizotinib $250 \mathrm{mg}$ twice a day in March 2019 in view of unresectable metastatic disease. Repeat CT chest done in June 2019 showed a complete response (CR) with resolution of lung lesions (Figure 1d). She has completed one year of therapy and continues to be in CR.

\section{Discussion}

IMTs are uncommon lung neoplasms representing around 1\% of all lung tumors [8]. Lung as the primary site has been described



Figure 1. Axial CT scan at baseline (a) in 2017 show well defined heterogeneously enhancing mass in left upper lobe (asterisk) with no fat or calcification within. The mass shows maintained fat planes with main pulmonary artery trunk and there is no extra-thoracic extension. Lung window of same point CT reveals no lung parenchymal or pleural nodules (b). Follow up CT scan after 2 years (c) of surgical resection showed tiny irregular parenchymal nodule in right lung and two pleural based deposits (arrows) which show complete resolution after 3 months of Crizotinib (d). 
almost as often as the abdomen and can present with constitutional symptoms in $15-30 \%$ with elevated proinflammatory markers. Our patient had history of low-grade fever and decreased appetite prior to developing cough. Fever is uncommon in primary lung carcinomas and hence may raise the suspicion of an alternate possibility [4]. IMTs of lung usually present as solitary pulmonary nodules (SPN) with lower lobe predilection without pleural or mediastinal invasion, however patterns at recurrence are less well defined [9]. Involvement of pleura is rare and has only been described in few case reports $[10,11]$. Our patient had lower lobe SPN initially but developed pleural nodules at recurrence which is rare.

Discovery of rearrangements in ALK gene on chromosome 2 p23 has revolutionized the understanding and management of IMT.


Figure 2. a) Low power photomicrograph of the tumor showing a spindle cell tumor with admixture of inflammatory cells. The tumor cells are arranged in short fascicles as well as haphazard pattern. b) High power photomicrograph showing tumor cells showing spindled morphology with finely dispersed chromatin and eosinophilic cytoplasm. There is abundant admixture of lymphocytes, plasma cells and few scattered eosnophils. c) Immunostain for smooth muscle actin showing cytoplasmic positivity. d) Immunostain for ALK-1 showing cytoplasmic positivity.
The ALK gene encodes a receptor tyrosine kinase which when constitutively activated owing to molecular rearrangement can give rise to tumorigenesis. Rearrangements in ALK can be detected in $50 \%$ of IMT by either fluorescence in-situ hybridization (FISH) or staining by IHC [12]. The prevalence of ALK positivity depends on primary tumor location (45\% lung, $60 \%$ GI, $62-71 \%$ bladder, $100 \%$ in peritoneum) [13]. Our patient showed diffuse positivity of ALK on IHC and hence, was a candidate for treatment with ALK inhibitors. Owing to activity of ALK inhibitors like crizotinib in ALK rearranged non-small cell lung cancer (NSCLC), they were tested in IMT as well. In the first published case series, two patients (one ALK positive and one negative) with advanced abdominal IMT were treated with crizotinib. The ALK positive patient attained a sustained CR (no toxicity) with no response in ALK negative patient, thus providing early evidence of biomarker based therapeutic strategy (14). On the basis of this data, a phase II trial was undertaken in Europe in which patients with unresectable/metastatic IMT were treated with crizotinib. ALK positivity was considered if $>15 \%$ cells showed diffuse staining for ALK on IHC. Out of 12 ALK positive patients, 6 patients achieved a response $(50 \%$ response rate) with two durable complete responses and four partial responses [15]. Among the ALK positive cohort, $73 \%$ patients were progression free at one year. Median duration of response was nine months with some patients achieving sustained remissions $(35 \%$ on treatment at median follow up of 2.3 years). This trial established crizotinib as treatment of choice for unresectable IMT. We started our patient on 250 mg BD of crizotinib. She developed complete radiological response after three months of starting therapy and is maintaining that response after 12 months of therapy at last follow up (duration of response- not reached). In line with advancements in field of molecular pathology, additional abnormalities have now been characterized in IMTs including ROS1 and RET gene fusions. In a recent case series of 62 cases of IMT, 35 patients (56\%) were positive for ALK, 6 patients $(10 \%)$ positive for ROS1 and one patient had RET gene arrangement [16]. Authors noted that fusion positive cases were more often reported in children and lug and soft tissue IMT's were most often positive for ALK or ROS1 fusions (83\% cases). Novel RET gene rearrangement was identified in pulmonary IMT. In a recent case report, Li et al. described partial response to ceritinib in a woman with ROS1 fusion positive IMT thus exploring yet another treatment paradigm in this extremely rare disease [17].

\section{Conclusions}

Precision oncology and targeted therapy has opened up novel avenues in the treatment of this extremely rare sarcoma. While crizotinib is now established as a standard of care for patients harboring ALK rearrangements, research into further molecular characterization holds promise for multiple other targeted therapies in future. However, literature on use of targeted agents in IMT from Indian subcontinent is scarce. This is the first report of complete response of ALK positive IMT to crizotinib from the Indian subcontinent and authors consider it as the first line of management in this disease.

\section{References}

1. Brunn $\mathrm{H}$. Two interesting benign lung tumors of contradictory histopathology. J ThoracSurg 1939;9:119-31.

2. Meis JM, Enzinger FM. Inflammatory fibrosarcoma of the 
mesentery and retroperitoneum. A tumor closely simulating inflammatory pseudotumor. Am J Surg Pathol 1991;15:1146- 56.

3. Coffin CM, Watterson J, Priest JR, Dehner LP. Extrapulmonary inflammatory myofibroblastic tumor (inflammatory pseudotumor). A clinicopathologic and immunohistochemical study of 84 cases. Am J Surg Pathol 1995;19:859-72.

4. Cook JR, Dehner LP, Collins MH, et al. Anaplastic lymphoma kinase (ALK) expression in the inflammatory myofibroblastic tumor: a comparative immunohistochemical study. Am J SurgPathol 2001;25:1364-71.

5. Saxena S, Dhal I, Mohanpuria A, et al. Extrapulmonary inflammatory myofibroblastic tumor at different sites with histopathology and immunohistochemical analysis: A case series. Oncol J India 2018;2:80-5.

6. Ray A, Suri JC, Bhattacharya D, Gupta A. Bronchoscopic resection of endobronchial inflammatory myofibroblastic tumor: A case report and systematic review of the literature. Lung India 2014;31:172-5.

7. Maturu VN, Bal A, Singh N. Inflammatory myofibroblastic tumor of the lung in pregnancy mimicking carcinoid tumor. Lung India 2016;33:82-4.

8. Cerfolio RJ, Allen MS, Nascimento AG, et al. Inflammatory pseudotumors of the lung. Ann ThoracSurg 1999;67:933-6.

9. Agrons GA, Rosado-de-Christenson ML, Kirejczyk WM, et al. Pulmonary inflammatory pseudotumor: radiologic features. Radiology 1998;206:511-8.

10. Dongel I, Yazkan R, Duman L, et al. Huge inflammatory myofibroblastic tumor of pleura with concomitant nuchal fibroma. Ann Thorac Surg 2013;96:1461-4.
11. Ueno T, Yamashita M, Sawada S, et al. A rare case of inflammatory myofibroblastic tumor of the diaphragmatic parietal pleura with dissemination. Acta Med Okayama 2015;69:65-8.

12. Coffin CM, Patel A, Perkins S, et al. ALK1 and p80 expression and chromosomal rearrangements involving 2p23 in inflammatory myofibroblastic tumor. Mod Pathol 2001;14:569-76.

13. Fuehrer NE, Keeney GL, Ketterling RP, et al. A protein expression and ALK gene rearrangements aid in the diagnosis of inflammatory myofibroblastic tumors of the female genital tract. Arch Pathol Lab Med 2012;136:623-6.

14. Butrynski JE, D'Adamo DR, Hornick JL, et al. Crizotinib in ALK-rearranged inflammatory myofibroblastic tumor. N Engl J Med 2010;363:1727-33.

15. Schöffski P, Sufliarsky J, Gelderblom H, et al. Crizotinib in patients with advanced, inoperable inflammatory myofibroblastic tumours with and without anaplastic lymphoma kinase gene alterations (European Organisation for Research and Treatment of Cancer 90101 CREATE): a multicentre, singledrug, prospective, non-randomised phase 2 trial. Lancet Respir Med 2018;6:431-41.

16. Antonescu CR, Suurmeijer AJ, Zhang L, et al. Molecular characterization of inflammatory myofibroblastictumors with frequent ALK and ROS1 gene fusions and rare novel RET rearrangement. Am J Surg Pathol 2015;39:957-67.

17. Li Y, Chen X, Qu Y, Fan JM, Li Y, Peng H et al. Partial response to ceritinib in a patient with abdominal inflammatory myofibroblastictumor carrying a TFG-ROS1 fusion. J Natl Compr Canc Netw 2019;17:1459-62. 